\title{
ESTUDO DE PROPRIEDADES ÓPTICAS DE FILME FINO DE ÓXIDO ANÓDICO DE TITÂNIO POR ELIPSOMETRIA *
}

\author{
Michele de Almeida Oliveira ${ }^{1}$ \\ Nayara Ferreira Nunes ${ }^{2}$ \\ Paulo Roberto da Silva² \\ Caroline da Silva Terra ${ }^{3}$ \\ Elivelton Alves Ferreira ${ }^{4}$ \\ Thomás Nascimento Caldeira Oliveira ${ }^{5}$ \\ Ladário da Silva ${ }^{6}$
}

\section{Resumo}

Neste presente estudo foi utilizada a técnica de elipsometria espectroscópica a fim de se caracterizar as propriedades ópticas do filme fino de óxido anódico de Titânio formado espontaneamente. As propriedades desse óxido são relevantes consideradas as diversas aplicações do titânio, dentre as quais as biológicas. A elipsometria é uma técnica não destrutiva, porém indireta, apropriada para obtenção de propriedades ópticas e dielétricas, após modelagem. Usando essa técnica, obtivemos além das propriedades ópticas, a espessura do óxido formado de forma natural sobre o substrato de Titânio.

Palavras-chave: Titânio; Óxido de titânio; Elipsometria

\section{STUDY OF THE OPTICAL PROPERTIES OF THE THIN FILM OF TITANIUM ANODIC OXIDE BY ELLIPSOMETRY}

\begin{abstract}
In this present study, the spectroscopic ellipsometry technique was used in order to characterize the optical properties of the spontaneous titanium anodic oxide thin film. The properties of this oxide are relevant considering the various applications of titanium, among which the biological ones. Ellipsometry is a non-destructive, but indirect, technique suitable for obtaining optical and dielectric properties, after modeling. Using this technique, we obtained besides the optical properties, the thickness of the oxide formed of natural form on the substrate of Titanium.

Keywords: Titanium; Titanium oxide; Ellipsometry.

1 Engenheira Produção, Mestranda em Engenharia Metalúrgica, Programa de Pós-Graduação em Engenharia Metalúrgica (PPGEM), Escola de Engenharia Industrial Metalúrgica de Volta Redonda (EEIMVR), Universidade Federal Fluminense (UFF), Volta Redonda, RJ - Brasil.

2 Engenheira Metalúrgica, Mestranda em Engenharia Metalúrgica, PPGEM, EEIMVR, UFF, Volta Redonda, RJ - Brasil.

3 Engenheira Mecânica, Mestranda em Engenharia Mecânica, PGMEC, EEIMVR, UFF, Volta Redonda, RJ-Brasil.

4 Doutor(a) em Química, Professor, Departamento de Química, Instituto de Ciências Exatas (ICEx), UFF, Volta Redonda, RJ-Brasil.

5 Biologo, Universidade Geraldo Di Biase (UGB).

6 Físico, Doutor em Física, Professor, ICEx, UFF, Volta Redonda, RJ - Brasil.
\end{abstract}




\section{INTRODUÇÃO}

O Titânio (Ti) é um elemento abundante na crosta terrestre, embora de difícil obtenção [1,2]. É muito utilizado em diversas áreas como por exemplo na fabricação de peças para motores, na fabricação de foguetes e produtos aeroespaciais, na produção de catalisadores para reações de polimerização de compostos organometálicos, na produção de ligas com outros metais, na produção de tintas e papeis, e em especial em diversas aplicações área biomédica. As principais reservas de minérios de titânio (rutilo, ilmenita e anatásio) do mundo estão localizadas na Noruega, Austrália, Canadá, Estados Unidos e Índia [3,4]. Sua biocompatibilidade se deve em parte a sua inatividade biológica e sua grande resistência à corrosão, que limita a quantidade de íons de titânio capazes de migrar para o tecido em contato [5]. Outras características de destaque são sua baixa densidade, sua boa resistência mecânica à tração, opacidade, inércia química, alta capacidade de dispersão e alto ponto de fusão. O titânio é muito utilizado na forma de oxido, em parte devido ao alto índice de refração. O titânio comercialmente puro é obtido a partir do rutilo e da ilmenita que são encontrados mais facilmente [6]. Alguns dos minérios de titânio são a ilmenita $\left(\mathrm{FeTiO}_{3}\right)$, o leucoxenio $(60 \% \mathrm{Tio2}$ e os $40 \%$ é material amorfo com e mistura do rutilo com anatásio), o rutilo $\left(\mathrm{TiO}_{2}\right)$ e o anatásio (TiO2) [4]. O anatásio quando aquecido acima de $915^{\circ} \mathrm{C}$ é convertido em rutilo[7,8].

Neste trabalho foi utilizada a técnica de elipsometria espectroscópica a fim de estudarmos a caracterização óptica do óxido anódico de titânio formado espontaneamente e do substrato de $\mathrm{Ti}[9,10]$. A elipsometria é uma técnica adequada para a caracterização óptica de superfícies, uma vez que é capaz de fornecer o índice de refração e o coeficiente de extinção em função do comprimento de onda [11,12]. Para isso ela precisa obter os parâmetros elipsométricos mudança de fase $(\Delta)$ e amplitude $(\Psi)$ em função do comprimento de onda incidente $\lambda[13,14]$. Esses parâmetros são funções dos componentes da camada de óxido e do substrato da amostra. Com os dados e realizando a modelagem dos mesmos $[15,16]$, é possível se obter a espessura da camada de óxido e as propriedades ópticas anteriormente citadas $[17,18]$.

\section{MATERIAL E MÉTODOS}

Para o presente trabalho foram utilizadas amostras de titânio (grau II), adquiridas pela UFF da Titânio Brasil em formato aproximado de um trapézio (vide figura 1). 


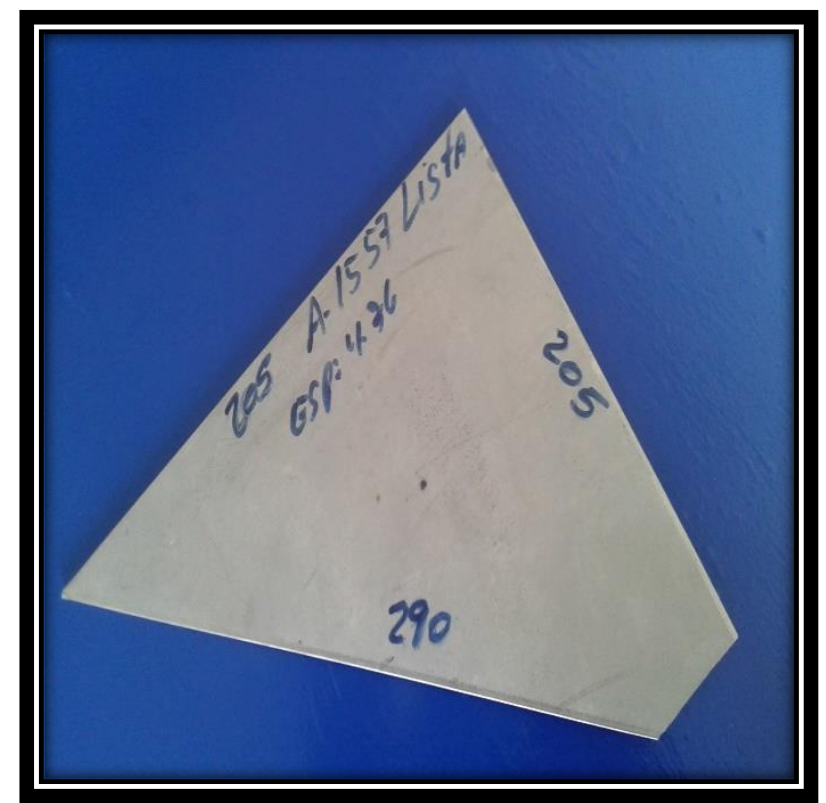

Figura 1. Exemplo de amostra de titânio metálico comprada na Titânio Brasil.

Em primeiro lugar a amostra foi cortada no laboratório de metalografia da Escola de Engenharia Industrial Metalúrgica de Volta Redonda (UFF-EEIMVR). Foram preparadas dez amostras identificadas individualmente. Em seguida as amostras foram embutidas em baquelite (vide figura 2), a fim de se realizar uma preparação metalográfica (lixamento e polimento) no titânio, pois para o uso da técnica de elipsometria é importante uma baixa rugosidade superficial. A amostra recebida possuía superfície irregular como exibido na figura 3 , obtida com o auxílio de um Microscópio Confocal Interferométrico LEICA, modelo DCM3D no Instituto de Ciências Exatas (ICEx) da UFF. O lixamento foi realizado em Politriz Lixadeira Metalografica Dupla Teclago, modelo PL02E. As lixas usadas, na sequência de granulometria, foram: 80, 120, 320, 400, 600, 800, 1000 e 1200 mesh. O polimento subsequente foi realizado com pano de polimento metalográfico, pasta de diamante e óleo apropriado. A rugosidade superficial foi monitorada antes de iniciar a preparação metalográfica (figura 3) e após o lixamento e polimento. Os valores da rugosidade superficial após o polimento foram obtidos e a rugosidade média das amostras foi de $0,3 \mu \mathrm{m}$.

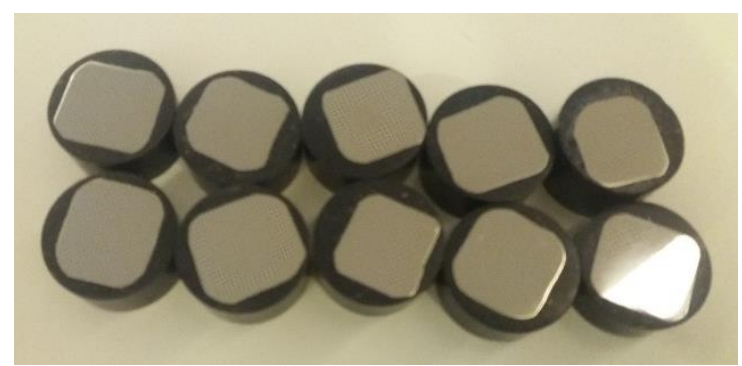

Figura 2. Amostras de Titânio metálico embutido em baquelite.

Ao fim do processo, as amostras foram lavadas com água ultrapura obtida de um dispositivo Milli-Q e álcool isopropílico. Em seguida foram secadas com um jato de ar quente. Imediatamente após, as mesmas foram medidas no elipsômetro espectroscópico SEMILAB GES 5S no ICEx da UFF (figura 4). 

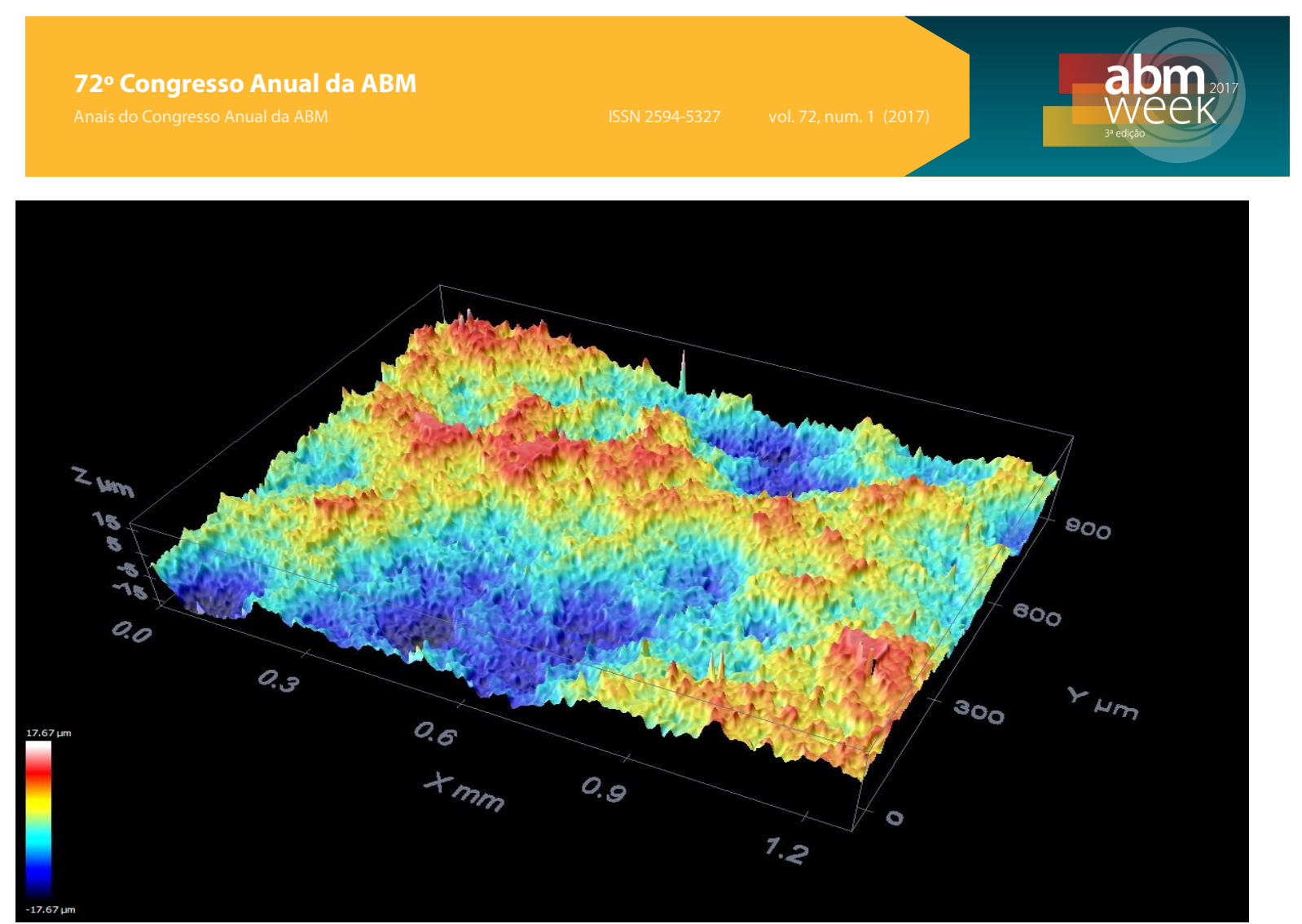

Figura 3. Topografia de superfície de amostra titânio como recebida.

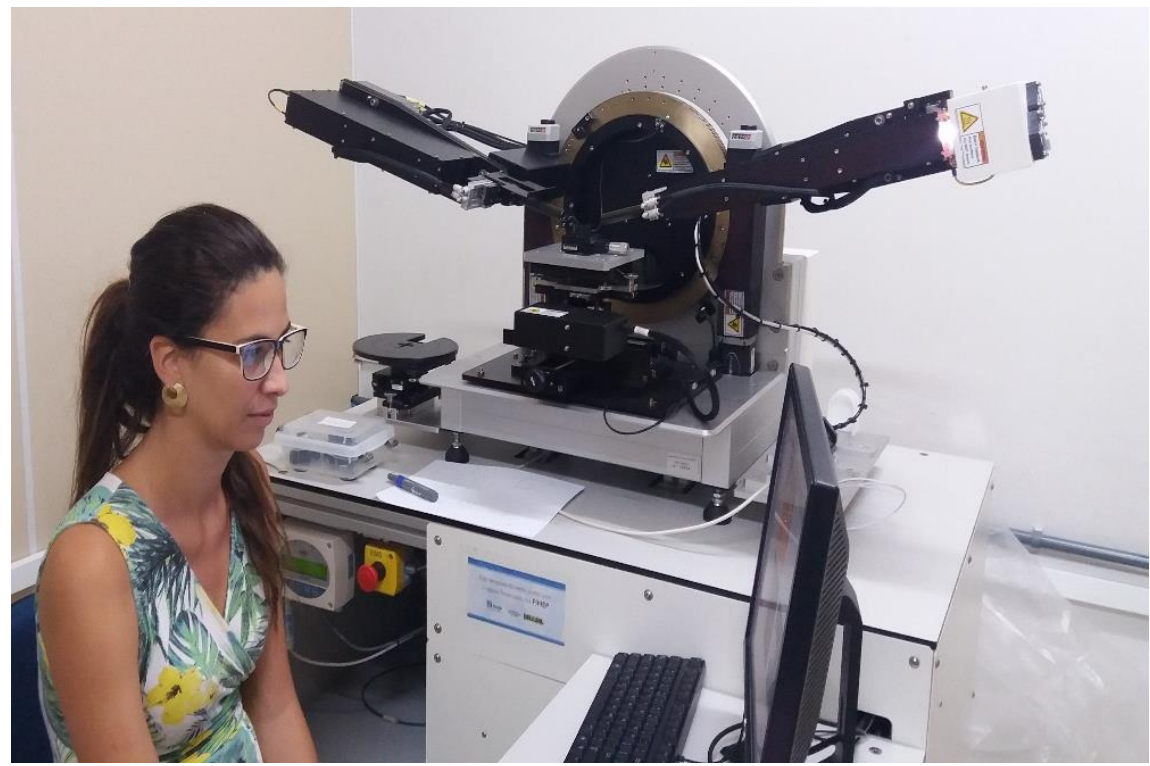

Figura 4. Elipsômetro Espectroscópico SEMILAB GES 5S

O elipsômetro espectroscópico utilizado possui lente de focalização (microspot), permitindo analisar áreas pequenas da ordem de 1,0 mm2. Neste experimento foram realizadas medidas com de $65^{\circ}, 70^{\circ}$ e $75^{\circ}$.

Para a realização da modelagem, adotou-se um modelo simples para a formação de óxido anódico de óxido de titânio sobre o substrato de titânio. A rugosidade foi considerada no modelo. 


\section{RESULTADOS E DISCUSSÃO}

Apresentamos um resultado típico para uma das amostras. Após a medida dos parâmetros elipsométricos $\tan (\Psi)$ e $\cos (\Delta)$, realizamos a modelagem e obtivemos um coeficiente de $R^{2}=0.98211$. Desta forma obtivemos as propriedades ópticas como índice de refração $(n)$ e coeficiente de extinção $(k)$, exibidos nas figuras 5 e 6 respectivamente $[19,20]$ para a modelagem correspondente ao $\mathrm{R}^{2}$ mencionado. Este resultado sugere que a técnica de medida e modelagem estão adequadas para continuarmos nas demais caracterizações. Conseguimos então medir a espessura do oxido no substrato com essa técnica, obtendo um valor de espessura para a camada de óxido de 13,8 nm.

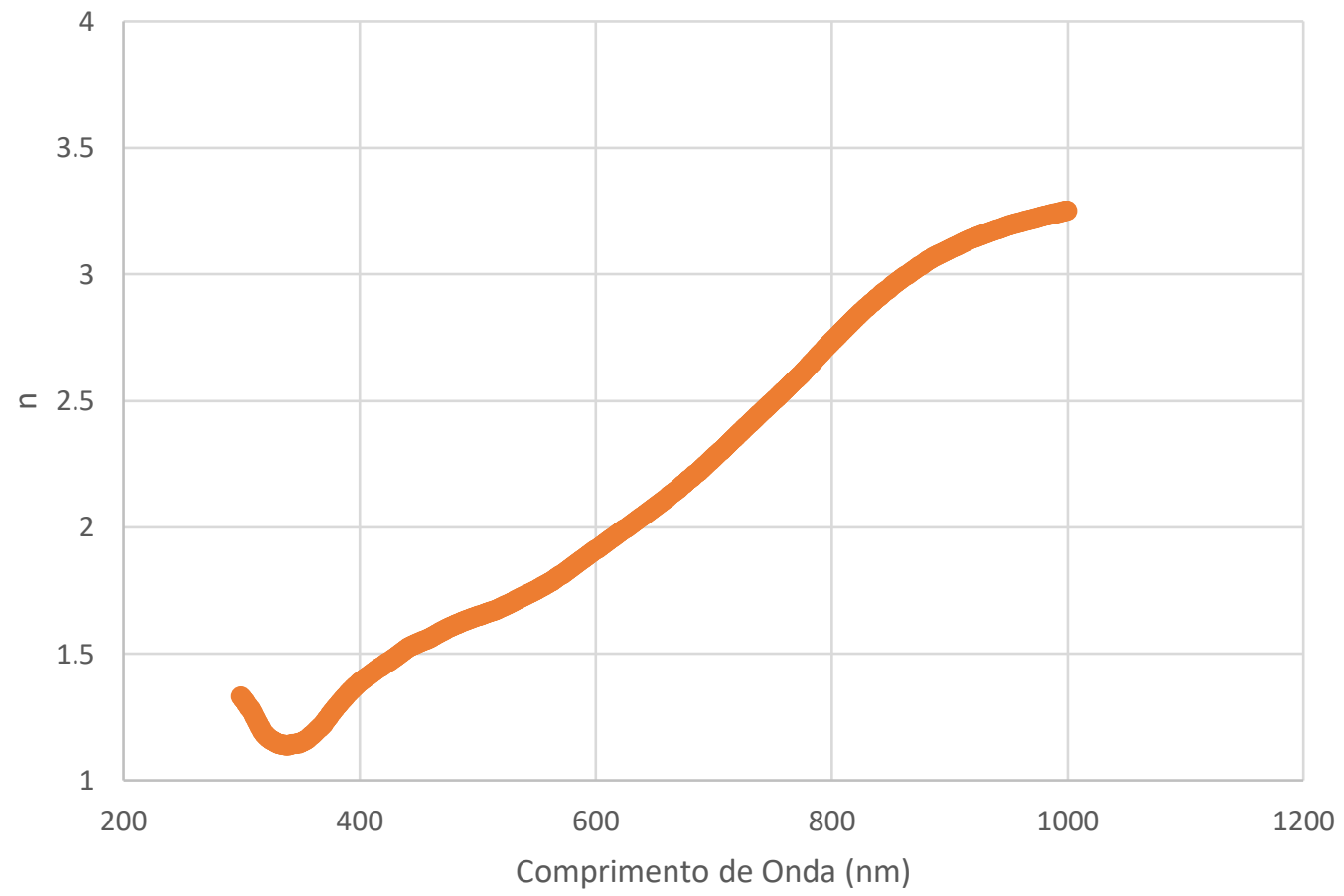

Figura 5. Índice de refração (n) em função do comprimento de onda. 


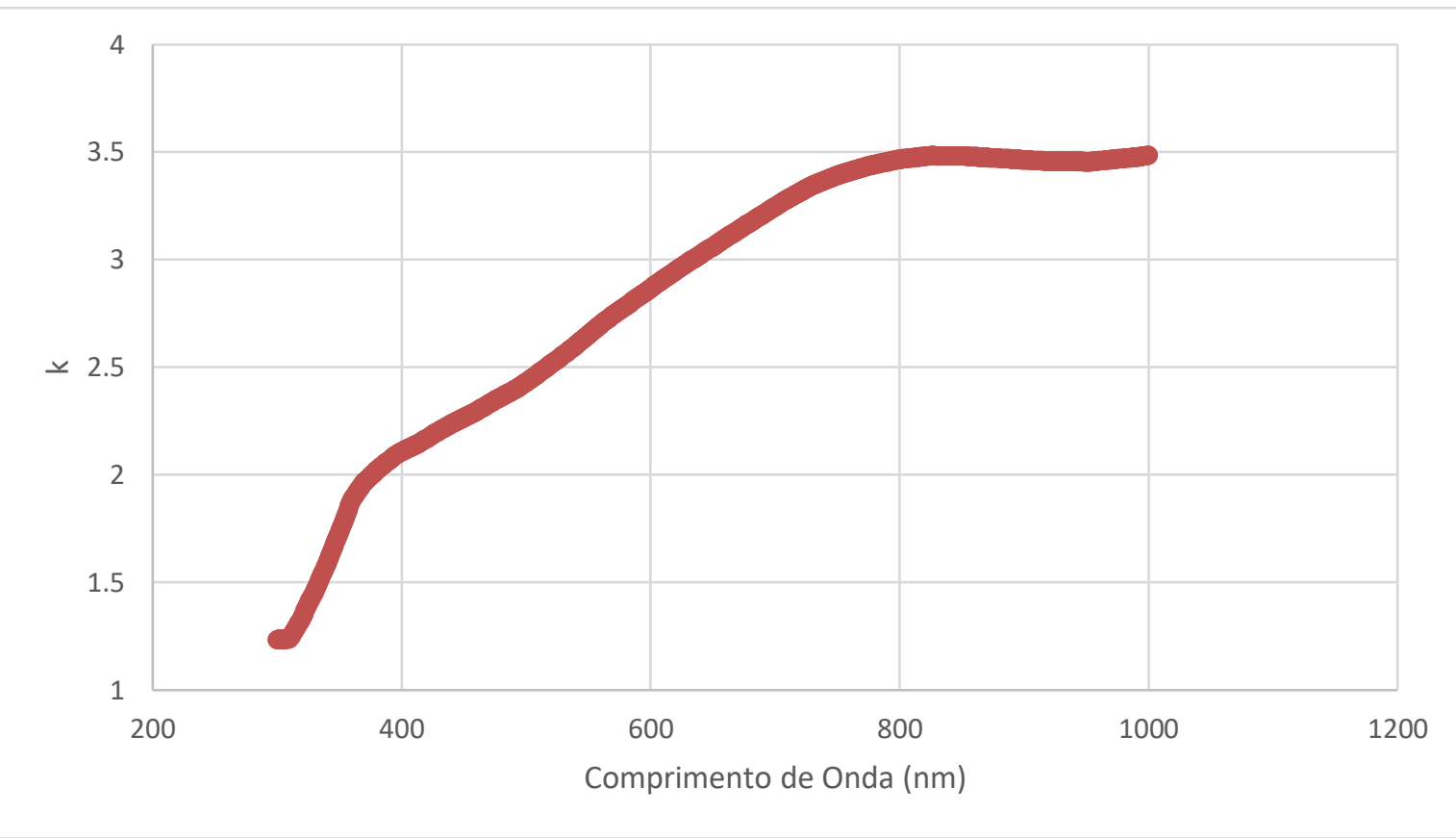

Figura 6. Coeficiente de extinção (k) em função do comprimento de onda.

As demais amostras apresentam resultados similares por todas terem passado pelo mesmo processo de lixamento e polimento. $\mathrm{Na}$ próxima etapa do estudo cresceremos óxido de titânio de forma controlada com espessuras diferentes e obteremos as propriedades ópticas e espessuras das diferentes amostras.

\section{CONCLUSÃO}

Neste presente estudo foi utilizada a técnica de elipsometria espectroscópica a fim de se caracterizar o substrato de Titânio e o filme fino de óxido anódico de Titânio formado espontaneamente. Através da correta modelagem, obtivemos a espessura do óxido, além do índice de refração e do coeficiente de extinção da camada de óxido. O modelo utilizado apresentou satisfatória equivalência com os dados experimentais, permitindo a continuidade dos estudos para a caracterização de óxidos crescidos controladamente, o qual iremos caracterizar.

\section{Agradecimentos}

Agradecemos à FINEP pela aquisição do Elipsômetro e Microscópio Confocal e à PROPPI-UFF pelo projeto FOPIN 2015. 


\section{REFERÊNCIAS}

1 Macedo ARH. Efeito do tratamento térmico do titânio sobre a proliferação de células pro-osteoblasticas. Dissertação do Mestrado.UFRN-PPGCEM.Natal.2008.

2 Bauer ORJ. Propriedades mecânicas do titânio comercialmente puro e da liga Ti-6Al$4 \mathrm{v}$ fundidos em diferentes ambientes. Dissertação do Doutorado. Universidade de São Paulo. USP. São Paulo.2007.

3 Ibris N, Rosca MCJ. EIS study of Ti and its alloys in biological media. Journal of Electroanalytical Chemistry. 2002; 526: 53-62.

4 Neto AAA, Almeida LBA. Titânio. Sumário Mineral Brasileiro.2008 [acesso em março 2017] Disponível em: http://www.dnpm.gov.br/dnpm/paginas/balancomineral/arquivos/balanco- mineral- brasileiro-2008-titanio.

5 Hanawa T. A comprehensive review of techniques for biofunctionalization of titanium. J Periodontal Implant Sci. 2011;41:263-272.

6 Benea L, Dănăilă $\mathrm{E}$, Ponthiaux $\mathrm{P}$. Porous TiO2-ZrO2 thin film formed by electrochemical technique to improve the biocompatibility of titanium alloy in physiological environment. IOP Conf. Series: Materials Science and Engineering. 2017;174:012044.

7 Sansiviero TM, Faria ALD. Influência do tratamento térmico no nanocompósito fotocatalisador ZnO/TiO2. Quim. Nova. 2014; Vol. XY, No. $00: 1-5$.

8 Stroppa GD, Giraldi RT, Leite RE, Varela AJ, Longo E. Obtenção de filmes finos de tio2 nanoestruturado pelo método dos precursores poliméricos. Quim. Nova.2008; Vol. 31, No. $7: 1706-1709$.

9 Junior AJ, Petri SFD, Maia BCF, Miranda BP. Filmes ultrafinos de esteres de celulose: Preparo, caracterização e imobilização de proteínas. Quim. Nova. 2010. Vol. 33, No. 10, 2064-2069.

10 Azevedo GGC. Caracterização óptica e eletrônica de filmes semicondutores usando espectros de transmitância e refletância. Universidade Estadual Paulista "Júlio de mesquita filho". Programa de Pós-graduação em Ciência e Tecnologia de Materiais. 2015

11 Gonçalves D, Irene AE. Fundamentals and applications of spectroscopic ellipsometry. Quim. Nova. 2002. Vol. 25, No. 5, 794-800.

12 Fernandes PRV. Caracterização de Guias de Ondas Por Elipsometria. Universidade de Aveiro Departamento de Física, 2010.

13 Kreling A, Silva L. Efeito da espessura e da temperatura de tratamento térmico nas propriedades ópticas e morfológicas de filmes finos de nitreto de silício. $8^{\circ}$ Congresso Brasileiro de Engenharia de Fabricação. 2015.

14 Vedam K, Spectroscopic Ellipsometry: a historical overview. Thin Solid Films.1998. 313314, 1 - 9.

15 Csermely Z, Horvath Z, Hanyecz I, Lugosi L. Spectroscopic Ellipsometry Analyzer - SEA User's Reference Manual. Semilab Co, Budapest, Hungary, 2012.

16 Aspnes DE, Spectroscopic ellipsometry - Past, present, and future. Thin Solid Films.2014. 571(3): 334-344.

17 Fujiwara H. Spectroscopic Ellipsometry: Principles and Applications. Japanese Edition, Tokyo, John Wiley \& Sons Ltd, 2003.

18 Johnson PB, Christy RW. Optical constants of transition metals: Ti, V, Cr, Mn, Fe, Co, $\mathrm{Ni}$, and Pd. Physical Review B, Volume 9, Number $12,1974$.

19 Januário LA. Avaliação da espessura e contaminantes da camada de óxido de titânio de diferentes marcas comerciais de implantes dentais osseointegraveis, analise por 
espectroscopia de fotoelétrons excitada por raios-x(xps). Faculdade de odontologia de Piracicaba. Unicamp. Piracicaba. 2001. 